\title{
Front Matter: Volume 8287
}

, "Front Matter: Volume 8287," Proc. SPIE 8287, Eighth Symposium Optics in Industry, 828701 (4 November 2011); doi: 10.1117/12.917126

SPIE. Event: Eighth Symposium Optics in Industry, 2011, Toluca de Lerdo, Mexico 


\title{
PROCEEDINGS OF SPIE
}

\section{Eighth Symposium Optics in Industry}

\author{
Eric Rosas \\ Norberto Arzate \\ Ismael Torres \\ Juan Sumaya \\ Editors
}

9-10 September 2011

Toluca de Lerdo, Estado de México, Mexico

Sponsored by

Academia Mexicana de Óptica (Mexico)

División de Óptica de la Sociedad Mexicana de Física (Mexico)

ICO Mexico Territorial Committee for Optics

Red Iberoamericana de Óptica

Organized by

Universidad Autónoma del Estado de México (Mexico)

Published by

SPIE 
The papers included in this volume were part of the technical conference cited on the cover and title page. Papers were selected and subject to review by the editors and conference program committee. Some conference presentations may not be available for publication. The papers published in these proceedings reflect the work and thoughts of the authors and are published herein as submitted. The publisher is not responsible for the validity of the information or for any outcomes resulting from reliance thereon.

Please use the following format to cite material from this book:

Author(s), "Title of Paper," in Eighth Symposium "Optics in Industry", edited by Eric Rosas,

Norberto Arzate, Ismael Torres, Juan Sumaya, Proceedings of SPIE Vol. 8287 (SPIE, Bellingham, WA, 2011) Article CID Number.

ISSN 0277-786X

ISBN 9780819489340

Published by

SPIE

P.O. Box 10, Bellingham, Washington 98227-0010 USA

Telephone +1 3606763290 (Pacific Time) · Fax +1 3606471445

SPIE.org

Copyright (C) 2011, Society of Photo-Optical Instrumentation Engineers

Copying of material in this book for internal or personal use, or for the internal or personal use of specific clients, beyond the fair use provisions granted by the U.S. Copyright Law is authorized by SPIE subject to payment of copying fees. The Transactional Reporting Service base fee for this volume is $\$ 18.00$ per article (or portion thereof), which should be paid directly to the Copyright Clearance Center (CCC), 222 Rosewood Drive, Danvers, MA 01923. Payment may also be made electronically through CCC Online at copyright.com. Other copying for republication, resale, advertising or promotion, or any form of systematic or multiple reproduction of any material in this book is prohibited except with permission in writing from the publisher. The CCC fee code is $0277-786 \mathrm{X} / 11 / \$ 18.00$.

Printed in the United States of America.

Publication of record for individual papers is online in the SPIE Digital Library.

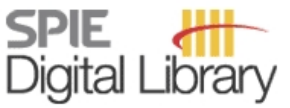

SPIEDigitalLibrary.org

Paper Numbering: Proceedings of SPIE follow an e-First publication model, with papers published first online and then in print and on CD-ROM. Papers are published as they are submitted and meet publication criteria. A unique, consistent, permanent citation identifier (CID) number is assigned to each article at the time of the first publication. Utilization of CIDs allows articles to be fully citable as soon as they are published online, and connects the same identifier to all online, print, and electronic versions of the publication. SPIE uses a six-digit CID article numbering system in which:

- The first four digits correspond to the SPIE volume number.

- The last two digits indicate publication order within the volume using a Base 36 numbering system employing both numerals and letters. These two-number sets start with 00, 01, 02, 03, 04, $05,06,07,08,09,0 A, 0 B \ldots 0 Z$, followed by 10-1Z, 20-2Z, etc.

The CID number appears on each page of the manuscript. The complete citation is used on the first page, and an abbreviated version on subsequent pages. Numbers in the index correspond to the last two digits of the six-digit CID number. 


\title{
Contents
}

\author{
ix Conference Committee \\ xi Introduction \\ xiii Conference Cosponsors
}

\section{OPTICS IN INDUSTRY}

828702 Optics in the nanoscale limit for optoelectronics and biophotonics [8287-68]

A. Neogi, Univ. of North Texas (United States)

828703 Optics industry in Spain: a cluster approach to increasing competitiveness through collaboration in R\&D and innovation [8287-43]

A. F. Cifuentes, Southern European Cluster in Photonics and Optics (Spain)

828704 Laser welding of nylon thin films using a pulsed $\mathrm{CO}_{2}$ waveguide laser [8287-03]

R. Villagomez, Ctr. de Investigación Científica y de Educación Superior en Ensenada Baja California (Mexico); R. Valenzuela, R. Camacho-Mesa, Poliflex, S.A. (Mexico)

828705 Analysis of incidence of keratoconus in relatives of patients who underwent corneal transplant due to advanced keratoconus using the Orbscan II topographic graphs [8287-04]

E. López-Olazagasti, Instituto Nacional Astrofísica Óptica Electronica (Mexico);

C. E. Hernández y del Callejo, Láser y Ultrasonido Ocular (Mexico); J. Ibarra-Galitzia,

G. Ramírez-Zavaleta, E. Tepichín Instituto Nacional de Astrofísica, Óptica y Electrónica (Mexico)

828706 Compound optofluidic small lenses with varifocal possibility [8287-05]

S. Calixto, Ctr. de Investigaciones en Óptica, A.C. (Mexico); M. Rosete Aguilar, CCADET, Univ. Nacional Autónoma de México (Mexico); O. L. Torres Rocha, F. J. Sanchez Marin, Ctr. de Investigaciones en Óptica, A.C. (Mexico); M. Calixto Solano, Tecnológico de Monterrey (Mexico); E. M. Martinez Prado, Ctr. de Investigaciones en Óptica, A.C. (Mexico)

828707 C-band optical fibers chromatic dispersion measurement at CENAM [8287-06]

Z. E. Ruíz, Ctr. Nacional de Metrología (Mexico); B. E. Ayala, Ctr. Nacional de Metrología (Mexico) and Ctr. de Investigaciones en Óptica, A.C. (Mexico); E. Rosas, Ctr. Nacional de Metrología (Mexico); I. Torres-Gómez, Ctr. de Investigaciones en Óptica, A.C. (Mexico); C. H. Matamoros García, Ctr. Nacional de Metrología (Mexico); J. A. Huerta-Ruelas, Instituto Politécnico Nacional (Mexico); N. Arzate, Ctr. de Investigaciones en Óptica, A.C. (Mexico)

828708 Effective detection plane location uncertainty component in luxmeters calibration [8287-08] R. López-Ramírez, L. P. González-Galván, A. Estrada-Hernández, E. Rosas, Ctr. Nacional de Metrología (Mexico) 
Judd-Ofelt analysis of the B-Te-Na-Si-Al:Er ${ }^{3+}$ polymolecular glass for IR broadband telecommunication [8287-10]

A. Lira, Univ. Autónoma del Estado de México (Mexico); I. Camarillo, Univ. Autónoma Metropolitana-Iztapalapa (Mexico); E. Camarillo, Univ. Nacional Autónoma de México (Mexico); U. Caldiño, Univ. Autónoma Metropolitana-Iztapalapa (Mexico); C. Falcony, Ctr. de Investigaciones del Instituto Politécnico Nacional (Mexico); G. H. Muñoz, Univ. Autónoma Metropolitana-Iztapalapa (Mexico); P. Rosendo, J. López, B. Ibarra, B. López, Univ. Autónoma del Estado de México (Mexico)

8287 OA Corrosion process of copper in chloride solution by optical interferometry [8287-11] G. Y. Enciso Soto, J. A. Marbán Salgado, Univ. Autónoma del Estado de Morelos (Mexico); O. Sarmiento Martínez, Technological Institute of Zacatepec (Mexico);

J. Uruchurtu Chavarin, D. Mayorga Cruz, Univ. Autónoma del Estado de Morelos (Mexico)

8287 OB Measurement of the absorption coefficient of a glucose solution through transmission of light and polarymetry techniques [8287-12]

J. Yáñez M., CIATEC, A.C. (Mexico)

8287 OC Comparison between quasi-sinusoidal and quasi-triangular profiles for diffraction gratings printed on acetates [8287-14]

M. Mora-González, R. Chiu-Zarate, J. Muñoz-Maciel, F. G. Peña-Lecona,

H. Pérez Ladrón de Guevara, F. J. Casillas, Univ. de Guadalajara (Mexico)

8287 OD Optical arrangement coupled to a radial shear cyclic interferometer to generate simultaneously two interferograms with a phase shift of $\Pi$ / 2 [8287-15]

J. F. Casco Vásquez, Instituto Tecnologico de Apizaco (Mexico) and Benemérita Univ. Autónoma de Puebla (Mexico); C. I. Robledo Sánchez, Benemérita Univ. Autónoma de Puebla (Mexico); M. Ortiz Gutiérrez, Univ. Michoacana de San Nicolás Hidalgo (Mexico); L. M. Arévalo Aguilar, Benemérita Univ. Autónoma de Puebla (Mexico);

P. Nanco Hernández, Benemérita Univ. Autónoma de San Nicolás Hidalgo (Mexico)

8287 OE Morphology of leaves cuticle by fringe projection [8287-19]

A. Martínez, J. A. Rayas Alvarez, Ctr. de Investigaciones en Óptica, A.C. (Mexico);

R. Cordero, Univ. de Santiago de Chile (Chile); D. Balieiro R., Univ. de Santiago de Chile (Chile), Leibniz Univ. Hannover (Germany), and Pontificia Univ. Católica de Chile (Chile)

8287 OF Polarimetric applications to identify bee honey [8287-20]

R. Espinosa-Luna, I. Saucedo-Orozco, C. V. Santiago-Lona, J. M. Franco-Sánchez, Ctr. de Investigaciones en Óptica, A.C. (Mexico); A. Magallanes-Luján, Univ. Autónoma de Zacatecas (Mexico)

8287 OG Monte Carlo method for evaluation of uncertainty in topometry by using in-plane electronic speckle pattern interferometry with divergent illumination [8287-21]

A. Martínez, Ctr. de Investigaciones en Óptica, A.C. (Mexico); J. Parra-Michel, Ctr. de Investigaciones en Óptica, A.C. (Mexico) and Univ. de La Salle Bajío (Mexico); R. Cordero, Univ. de Santiago de Chile (Chile); J. A. Rayas, Ctr. de Investigaciones en Óptica, A.C. (Mexico) 
$8287 \mathrm{OH} \quad$ Crystallinity and vinyl groups formation in polyethylene films exposed to UV-B radiation [8287-24]

A. Martínez-Romo, R. González-Mota, J. J. Soto-Bernal, Instituto Tecnológico de Aguascalientes (Mexico); C. Frausto-Reyes, Ctr. de Investigaciones en Óptica, A.C., (Mexico); I. Rosales-Candelas, E. I. Muñoz-Campos, Instituto Tecnológico de Aguascalientes (Mexico)

8287 Ol Modeling the tapering effects on the modal parameters of a hollow-core photonic bandgap fiber [8287-30]

N. González Baquedano, S. Vargas, N. Arzate, I. Torres-Gómez, A. Martínez-Ríos, D. E. Ceballos-Herrera, Ctr. de Investigaciones en Óptica, A.C. (Mexico); A. Ferrando, Univ. de Valencia (Spain); C. Milián, Univ. Politécnica de Valencia (Spain)

$8287 \mathrm{OJ} \quad$ Application of $\mathrm{CO}_{2}$ laser for electronic components soldering [8287-32] J. Mascorro-Pantoja, CICATA-IPN (Mexico); J. J. Soto-Bernal, Instituto Tecnológico de Aguascalientes (Mexico); M. Nieto-Pérez, R. Gonzalez-Mota, I. Rosales-Candelas, CICATA-IPN (Mexico)

8287 OK Digital podoscope for remote diagnosis [8287-33] A. A. Silva Moreno, CIATEC, A. C. (Mexico); F. Chávez Gutiérrez, Univ. Politécnica del Bicentenario (Mexico)

$8287 \mathrm{OL}$ Retrieval columns of $\mathrm{SO}_{2}$ in industrial chimneys using DOAS passive in traverse [8287-36] R. Galicia Mejía, J. M. de la Rosa Vázquez, Instituto Politécnico Nacional (Mexico); G. Sosa Iglesias, Instituto Mexicano del Petróleo (Mexico)

$8287 \mathrm{OM}$ Optical system for the supervision of the operation of a induction motor [8287-37] V. M. Villanueva-Reyes, R. A. Vazquez-Nava, Ctr. de Investigaciones en Óptica, A.C. (Mexico)

8287 ON Traffic infrastructure inventory system by analysis image [8287-39]

A. I. Rico-Martinez, J. C. Camarillo-Paz, F. J. Ornelas-Rodríguez, J.-J. Gonzalez-Barbosa, Y. C. Peña Cheng, J. B. Hurtado-Ramos, CICATA-IPN (Mexico)

$828700 \quad$ Multi-level optical memory based in $\mathrm{Ge}_{1} \mathrm{Sb}_{2} \mathrm{Te}_{4}$ [8287-40]

E. Morales-Sánchez, CICATA-IPN (Mexico); E. Prokhorov, CINVESTAV del IPN (Mexico);

C. Rivera-Rodríguez, ININ (Mexico); Yu. Kovalenko, CIATEQ (Mexico);

J. González Hernandez, CIMAV (Mexico)

8287 OP Evaluation of optical wireless communications systems by means of the turbulence chamber [8287-41]

J. de Dios Sánchez, J. I. Nieto, Univ. Autónoma de Baja California (Mexico); A. Arvizu, F. J. Mendieta, CICESE (Mexico); M. Vázquez, Univ. Autónoma de Baja California (Mexico)

$8287 \mathrm{OQ}$ Interaction of Gaussian beams with a conducting finite grating: transmission case [8287-45] M. G. Martínez-Flores, J. Sumaya-Martínez, G. Montiel-Gonzalez, J. C. Corona-Oran, Univ. Autónoma del Estado de México (Mexico); O. Mata-Méndez, Instituto Politécnico Nacional (Mexico) 
8287 OR Rigorous diffraction of Hermite-Gaussian beams by a double slit: TM polarization case [8287-46]

G. Enriquez-Leon, M. G. Martínez-Flores, G. Torres-Morales, J. Sumaya-Martínez, Univ. Autónoma del Estado de México (Mexico); S. Lopez-Lopez, Ctr. Nacional de Metrología (Mexico)

8287 OS Interaction of Gaussian beams with a conducting finite grating: reflection case [8287-47] G. Torres-Morales, J. Sumaya-Martínez, A. Pato-Córdoba, Univ. Autónoma del Estado de México (Mexico)

8287 OT Enhanced transmission through metallic subwavelength slits [8287-48]

G. F. Camacho-Gonzalez, Univ. Autónoma del Estado de México (Mexico); O. Olmos-Lopez, Tecnológico de Monterrey (Mexico); J. Sumaya-Martinez, M. Mayorga-Rojas, Univ. Autónoma del Estado de México (Mexico)

8287 OU Spatial location of reference points for the study in $360^{\circ}$ of an object using Stereo Vision [8287-49]

V. H. Flores, A. Martínez, J. A. Rayas, Ctr. de Investigaciones en Óptica, A.C. (Mexico);

K. Genovese, Univ. degli Studi della Basilicata (Italy)

8287 OV A genetic algorithm applied in the three-dimensional reconstruction of digitalized objects [8287-50]

D. Torres, F. J. Cuevas, Ctr. de Investigaciones en Óptica, A.C. (Mexico)

8287 OW Potential uses of a Mueller polarimeter in the industry [8287-52]

G. Martínez-Ponce, Ctr. de Investigaciones en Óptica, A.C. (Mexico)

8287 OX Design considerations and modifications on an optomechatronic load cell [8287-53] A. A. Camacho P., F. J. Martínez Serrano, O. M. García D., Univ. de la Salle Bajío (Mexico);

8287 OY Three beams phase-shifting interferometry by their amplitude variation [8287-54] C. Meneses-Fabian, U. Rivera-Ortega, Benémerita Univ. Autónoma de Puebla (Mexico)

$8287 \mathrm{OZ}$ Micro-displacement sensor using a Mach-Zehnder interferometer with long-period gratings [8287-57]

K. M. Salas-Alcántara, I. Torres-Gómez, D. Monzón-Hernández, A. Martínez-Ríos,

L. Armando García-de-la-Rosa, Ctr. de Investigaciones en Óptica, A.C. (Mexico)

828710 Automatic re-calibration by laser metrology and computer algorithms [8287-58] J. A. Muñoz Rodríguez, Ctr. de Investigaciones en Óptica, A.C. (Mexico);

E. F. Velazquez Pedroza, Univ. de Guadalajara (Mexico)

828711 Topometry and color association by RGB fringe projection technique [8287-60] Y. Y. López Domínguez, A. Martínez, J. A. Rayas, Ctr. de Investigaciones en Óptica, A.C (Mexico); K. Genovese, Univ. degli Studi della Basilicata (Italy)

828712 Fourier transform method to measure of the chromatic dispersion in an optical fiber [8287-62]

J. Ramos-Beltrán, G. Beltrán-Pérez, J. Castillo-Mixcóatl, S. Muñoz-Aguirre, Benemérita Univ. Autónoma de Puebla (Mexico) 
828713 Fringe pattern simulation with an adaptive pendulum [8287-63]

W. F. Guerrero-Sánchez, R. Juárez-Salazar, C. Meneses-Fabian, C. I. Robledo-Sánchez,

Benemérita Univ. Autónoma de Puebla (Mexico)

828714 Radial slope measurements of transparent samples using phase shifting interferometry by polarization [8287-65]

D.-I. Serrano-García, N.-I. Toto-Arellano, A. Martínez-García, Ctr. de Investigaciones en Óptica, A.C. (Mexico); G. Rodríguez-Zurita, Benemérita Univ. Autónoma de Puebla (Mexico)

828715 Ray tracing to study of waxes around the cloud point by optical absorption tomography [8287-67]

L. Moreno-Alvarez, C. Meneses-Fabian, J. N. Herrera, G. Rodríguez-Zurita, Benemérita Univ. Autónoma de Puebla (Mexico)

Author Index 
Proc. of SPIE Vol. $8287828701-8$

Downloaded From: https://www.spiedigitallibrary.org/conference-proceedings-of-spie on 26 Apr 2023 Terms of Use: https://www.spiedigitallibrary.org/terms-of-use 


\title{
Symposium Committees
}

\author{
Conference Chair
}

Juan Sumaya Martínez, Universidad Autónoma del Estado de México (Mexico)

National Advisory Council

Eduardo Gasca Pliego, Universidad Autónoma del Estado de México (Mexico)

Miguel Mayorga Rojas, Universidad Autónoma del Estado de México (Mexico)

Ramón Rodríguez Vera, Academia Mexicana de Óptica (Mexico)

Sergio Vázquez y Montiel, División de Óptica de la Sociedad

Mexicana de Física (Mexico)

Steering Committee

Alfonso Lastras Martínez, IICO, Universidad Autónoma de San Luis Potosí (Mexico)

Oracio C. Barbosa García, Centro de Investigaciones en Óptica, A.C. (Mexico)

Eduardo Tepichín Rodríguez, Instituto Nacional de Astrofísica, Óptica y Electrónica (Mexico)

Diana Tentori Santa Cruz, Centro de Investigación Científica y de Educación Superior de Ensenada (Mexico)

Eric Rosas, Centro Nacional de Metrología (Mexico)

Julio César Gutiérrez Vega, CO, Tecnológico de Monterrey (Mexico)

Guillermo García Torales, CUCEl, Universidad de Guadalajara (Mexico)

Program Committee

Amalia Martínez García, Academia Mexicana de Óptica (Mexico) and Centro de Investigaciones en Óptica, A.C. (Mexico)

Lorena Romero Salazar, Universidad Autónoma del Estado de México (Mexico) Norberto Arzate Plata, Centro de Investigaciones en Óptica, A.C. (Mexico)

Ismael Torres Gómez, Centro de Investigaciones en Óptica, A.C. (Mexico)

Scientific Committee

Daniel Malacara Hernández, AMO \& Centro de Investigaciones en Óptica, A.C. (Mexico)

Roberto Machorro Mejía, AMO \& CNyN, Universidad Nacional

Autónoma de México (Mexico)

Luis Efraín Regalado, AMO \& Universidad de Sonora (Mexico) 
J. Javier Sánchez Mondragón, AMO \& Instituto Nacional de Astrofísica, Óptica y Electrónica (Mexico)

Jorge Ojeda Castañeda, AMO \& DICIS, Universidad de Guanajuato (Mexico)

Miguel Torres Cisneros, AMO \& DICIS, Universidad de Guanajuato (Mexico)

Alfonso Lastras Martínez, AMO \& IICO, Universidad Autónoma de San Luis Potosí (Mexico)

Oracio C. Barbosa García, AMO \& Centro de Investigaciones en Óptica, A.C. (Mexico)

Fernando Mendoza Santoyo, AMO \& Centro de Investigaciones en Óptica, A.C. (Mexico)

Eric Rosas, AMO \& Centro Nacional de Metrología (Mexico)

Emmanuel Haro Poniatowski, DO-SMF \& Universidad Autónoma Metropolitana - Iztapalapa (Mexico)

Ramón Rodríguez Vera, DO-SMF \& Centro de Investigaciones en Óptica, A.C. (Mexico)

J. Rufino Díaz Uribe, DO-SMF \& CCADET, Universidad Nacional Autónoma de México (Mexico)

Fermín Salomón Granados Agustín, DO-SMF \& Instituto Nacional de Astrofísica, Óptica y Electrónica (Mexico) 


\section{Introduction}

In 2001, a group of very enthusiastic Mexican scientists in the field of optics and photonics visualized a forum were the Mexican researchers could transfer their knowledge into innovative industrial processes and products, in order to develop the optics and phonics industry in Mexico; this initiative crystallized in the symposium "Optics in Industry" (SOI).

Since then, the SOl has been successfully organized by the Instituto de Investigación en Comunicación Óptica of the Universidad Autónoma de San Luis Potosí (San Luis Potosí, 2001); the Centro de Investigaciones en Óptica A.C. (León, 2002), the Instituto Nacional de Astrofísica, Óptica y Electrónica (Puebla, 2003), the Centro de Investigación Científica y de Educación Superior de Ensenada (Ensenada, 2004), the Centro Nacional de Metrología (Santiago de Querétaro, 2005), the Centro de Óptica of the Instituto Tecnológico y de Estudios Superiores de Monterrey (Monterrey, 2007) and the Centro Universitario de Ciencias Exactas e Ingenierías of the Universidad de Guadalaja (2009).

In 2011 the Facultad de Ciencias of the Universidad Autónoma del Estado de México (UAEM) was honored as the host institution of the Eighth Symposium "Optics in Industry" (VIII SOI), which was held on September 9 and 10, in the city of Toluca de Lerdo. This eighth SOI edition allowed those who apply the optics and photonics technology to meet their developers and join their efforts towards the definition of strategic areas for development and towards the immediate solution of the problems in industry. Its main objective looks for the consolidation of the collaboration between industry and academy so that both benefit in the mean time by means of the promotion of agreements focused in this sense; and in the long term with the construction of a better World after the application of green optics and photonics technology.

On behalf of the Universidad Autónoma del Estado de México, the Academia Mexicana de Óptica, the División de Óptica de la Sociedad Mexicana de Física, the ICO Mexico Territorial Committee for Optics and the Red Iberoamericana de Óptica, we kindly acknowledge the attendees and sponsors for all your valuable participation and support to the Eighth Symposium "Optics in Industry" and hope you enjoyed your stay in Toluca de Lerdo.

Juan Sumaya Martínez 
Proc. of SPIE Vol. 8287 828701-12

Downloaded From: https://www.spiedigitallibrary.org/conference-proceedings-of-spie on 26 Apr 2023 Terms of Use: https://www.spiedigitallibrary.org/terms-of-use 


\section{SOI Sponsors}

Organized by

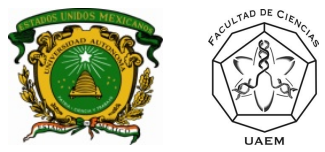

Coorganized by

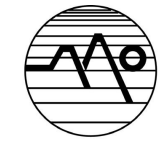

ACADEMIA MEXICANA
DE ÓPTICA, A.C.

SMF

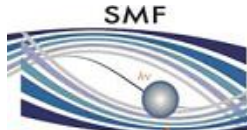

División Ōptica

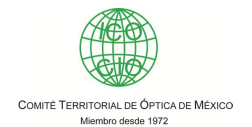

RIAO
Facultad de Ciencias,

Universidad Autónoma del Estado de México

Academia Mexicana de Óptica

División de Óptica, Sociedad Mexicana de Física

ICO Mexico Territorial Committe for Optics

Red Iberoamericana de Óptica

With the help of
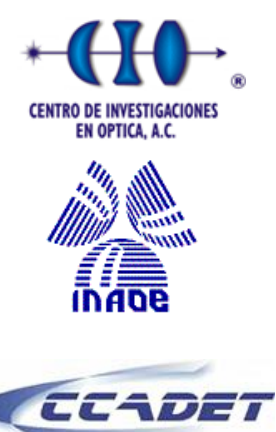

CENTRO DE CIENCInS APUCADAS Y
DESARBOLO TECMOLOGIO
Centro de Investigaciones en Óptica

Instituto Nacional de Astrofísica, Óptica y Electrónica

Centro de Ciencias Aplicadas y Desarrollo Tecnológico, Universidad Nacional Autónoma de México
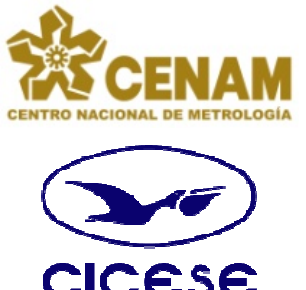

cicese

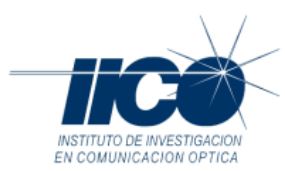

Centro Nacional de Metrología

Centro de Investigación Científica y de Educación Superior de Ensenada

Instiuto de Investigación en Comunicación Óptica, Universidad Autónoma de San Luis Potosí 


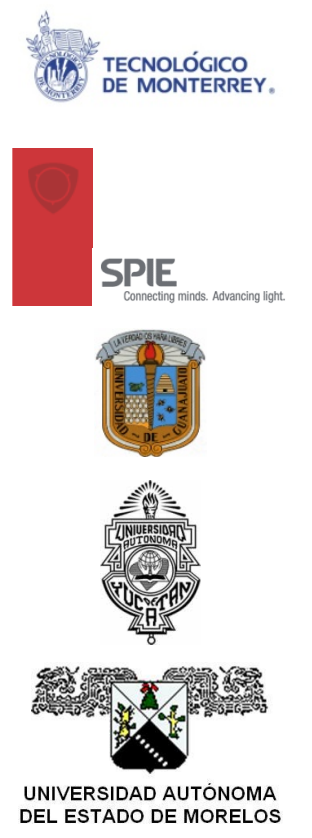

Centro de Óptica,

Tenológico de Monterrey

SPIE is the internacional society for optics and photonics

Universidad de Guanajuato

Universidad Autónoma de Yucatán

Universidad Autónoma del Estado de Morelos

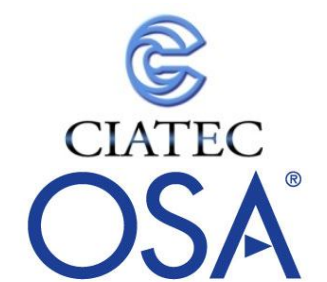

The Optical Society

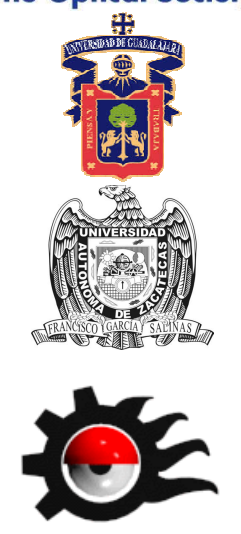

Centro de Innovación Aplicada en Tecnologías Competitivas

The Optical Society of America

Universidad de Guadalajara

Universidad Autónoma de Zacatecas

Centro de Investigación en Ciencia Aplicada y Tecnología Avanzada, Instituto Politécnico Nacional
TNATIONAL INSTRUMENTS

\section{مि TELMEX SATELSA Agilent Technologies<smiles>c1ccc2ccccc2c1</smiles>

National Instruments México, S. A. de C. V.

Teléfonos de México, S. A. de C. V.

Sistemas y Aplicaciones de Telecomunicaciones, S. A. de C. V.

Agilent Technologies México, S. de R. L de C. V. 
Acterna de México, S. A. de C. V.

\section{YOKOGAWA Supervy Sistemas, S. A. de C. V.}

\section{$\frac{d r}{12}$ \\ VARIAN \\ PHILIPS construlita}

\section{AF FLLR}

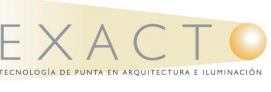

INSTRUMet

SA dec

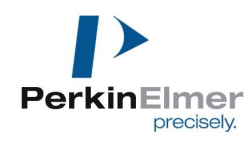

precisely.

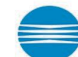

KONICA MINOLTA [19] METROCAL

e acolor
Insimet, S. A. de C. V.

Varian, S. de R. L. de C. V.

Construlita de Querétaro S. A. de C. V.

Sistemas Infrarrojos y de Potencia KLM, S. A.

Exacto Tecnología de Punta en Arquitectura e lluminación

Laboratorio de Calibración y Calificación S. A. de C. V.

Perkin Elmer de México S. A.

Metrocal S. A. de C. V.

Exacolor Laboratories, S. A. de C. V. 


\section{THOR】ABgS Thor Labs, Inc.}

\section{Mlultion}

QOcean

MIXBAAL
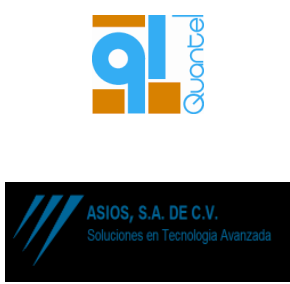

ग-Electrónica

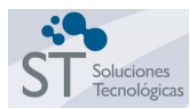

MultiON Consulting, S. A. de C. V.

Ocean Optics, México, S. A. de C. V.

MIXBAAL, S. A. de C. V.

Quantel Medical, S. A. de C. V.

Asios, S. A. de C. V.

DEC-Electrónica, S. A. de C. V.

Soluciones Tecnológicas, S. A. de C. V. 